\title{
5-Fluorouracil degradation rate as a predictive biomarker of toxicity in breast cancer patients treated with capecitabine
}

J Oncol Pharm Practice $0(0)$ I-7

(C) The Author(s) 2020 Article reuse guidelines: sagepub.com/journals-permissions DOI: $10.1177 / 1078155220904999$ journals.sagepub.com/home/opp @SAGE

\author{
Andrea Botticelli', Simone Scagnoli' (1), Michela Roberto², \\ Luana Lionetto ${ }^{2}$, Bruna Cerbelli ${ }^{2}$, Maurizio Simmaco ${ }^{3}$ and \\ Paolo Marchetti ${ }^{4}$
}

\begin{abstract}
Capecitabine is an oral prodrug of 5-fluorouracil with a relevant role in the treatment of breast cancer. Severe and unexpected toxicities related to capecitabine are not rare, and the identification of biomarkers is challenging. We evaluate the relationship between dihydropyrimidine dehydrogenase, thymidylate synthase enhancer region and methylenetetrahydrofolate reductase polymorphisms, 5-fluorouracil degradation rate and the onset of G3-4 toxicities in breast cancer patients. Genetic polymorphisms and the 5 -fluorouracil degradation rate of breast cancer patients treated with capecitabine were retrospectively studied. Genetic markers and the 5-fluorouracil degradation rate were correlated with the reported toxicities. Thirty-seven patients with a median age of 58 years old treated with capecitabine for stages II-IV breast cancer were included in this study. Overall, 34 (91.9\%) patients suffered from at least an episode of any grade toxicity while nine patients had G3-4 toxicity. Homozygous methylenetetrahydrofolate reductase 677TT was found to be significantly related to haematological toxicity (OR $=6.5$ [95\% IC I.I-37.5], P=0.04). Three patients had a degradation rate less than $0.86 \mathrm{ng} / \mathrm{mL} / 106$ cells $/ \mathrm{min}$ and three patients greater than $2.1 \mathrm{ng} / \mathrm{mL} / 106 \mathrm{cells} / \mathrm{min}$. At a univariate logistic regression analysis, an altered value of 5 -fluorouracil degradation rate (values $<0.86$ or $>2.10 \mathrm{ng} / \mathrm{mL} /$ 106 cells $/ \mathrm{min}$ ) increased the risk of $\mathrm{G} 3-4$ adverse events $(\mathrm{OR}=10.40$ [95\% IC: I.48-7.99], $\mathrm{P}=0.02)$. A multivariate logistic regression analysis, adjusted for age, comorbidity and CAPE-regimen, confirmed the role of 5-fluorouracil degradation rate as a predictor of $\mathrm{G} 3-4$ toxicity occurrence $(\mathrm{OR}=10.9$ [95\% IC I.2-96.2], $\mathrm{P}=0.03)$. The pre-treatment evaluation of 5 -fluorouracil degradation rate allows to identify breast cancer patients at high risk for severe 5-FU toxicity.
\end{abstract}

\section{Keywords}

5-FU degradation rate, breast cancer, capecitabine, chemotherapy toxicity, polymorphism

Date received: 26 May 2019; revised: I3 December 2019; accepted: I5 January 2020

\section{Introduction}

Capecitabine is an oral prodrug of 5-fluorouracil (5-FU), enzymatically activated by timidina phosphorylase in tumour tissue, that was rationally designed to mimic continuous infusion 5-FU. ${ }^{1}$ Capecitabine is rapidly absorbed from the gastrointestinal (GI) tract and metabolized by carboxylesterase in liver, and it is converted to $5^{\prime}$ deoxy-5-fluorocytidine and then to $5^{\prime}$ deoxy-5-fluorouridine (5' DFUR) by cytidine deaminase. Finally, the enzyme thymidine phosphorylase converts $5^{\prime}$ DFUR to 5-FU both in normal and tumour tissues. However, after an oral dose of capecitabine, the concentration of 5-FU in tumor tissue is higher than in adjacent healthy tissue, as result of an higher activity of Thymidine phosphorylase (TP). ${ }^{2}$ Oral administration seems to be preferred by patients and allows to avoid complications and costs linked to 5-FU IV infusion. ${ }^{3}$

\footnotetext{
'Azienda Policlinico Umberto I Roma, Roma, Italy

${ }^{2}$ Azienda Ospedaliera Sant'Andrea, Roma, Lazio, Italy

${ }^{3}$ Department of Neurosciences, Mental Health and Sensory Organs (NESMOS), Sapienza University of Rome, Roma, Italy

${ }^{4}$ Department of Medical Oncology, St Andrea University Hospital, Rome, Italy

Corresponding author:

Simone Scagnoli, Azienda Policlinico, Umberto I Viale del Policlinico, I55 Roma, Roma 00161, Italy.

Email: simone.scagnoli@hotmail.it
} 
In advanced breast cancer ( $A B C)$, the efficacy and safety of capecitabine have been demonstrated both as monotherapy and in combination with other drugs. Several studies have demonstrated the efficacy of capecitabine at the dose of 1250 or $1000 \mathrm{mg} / \mathrm{m}^{2}$ twice daily for 14 over 21 days in metastatic breast cancer patients. $^{4,5}$ The oral prodrug has also been tested in an older population of patients ( $>65$ years old) resulting effective and well tolerated at the dose of $1000 \mathrm{mg} / \mathrm{m}^{2}{ }^{6}$ Capecitabine is a landmark treatment also in HER2+ metastatic disease in association with lapatinib, after initial progression to first or second line. ${ }^{7}$

Conversely, the role of capecitabine in the adjuvant setting is still uncertain. Several trials of adjuvant capecitabine administered in combination with other effective drugs did not show an advantage over regimens without capecitabine. ${ }^{8,9}$ Recently, in the CRATE study, about 900 patients with HER2 negative breast cancer with residual disease after standard neoadjuvant chemotherapy have been randomized to receive adjuvant capecitabine or no treatment (control group). The results showed improvement in disease-free survival $(74.1 \%$ vs. $67.6 \%$ r at 5 years, respectively, HR 0.70 , p: 0.01$)$ and overall survival (OS) $(89.2 \%$ vs. $83.6 \%$ respectively, $\mathrm{HR} 0.59 ; \mathrm{P}=0.01)$ in treatment group and even more significant in TNBC subgroup. ${ }^{10}$ As expected, the addition of capecitabine caused treatment-related toxicities. ${ }^{11,12}$

Regardless cancer primary site and setting of treatment, however, capecitabine is related with several high grade toxicities, some of them with quick onset and unexpected severity. ${ }^{13}$ Diarrhea is the most common, adverse event that occurs in $55-60 \%$ of patients, with a severe grade 3 or 4 of toxicity in about $12-15 \%$. Other common GI adverse events are nausea and vomiting. Moreover, haematological adverse events like neutropenia, anaemia and thrombocytopenia are very common during the treatment. Finally, skin reactions are common: rash and hand\&foot syndrome are frequent but also severe skin toxicities such as StevensJohnson syndrome and toxic epidermal necrolysis are reported. ${ }^{14-16}$

In view of the wide use of capecitabine in breast cancer, the identification of predictive factors for capecitabine-related toxicities is a pressing need. Preemptive identification of patients that will develop severe toxicities is still an open issue. Several tests have been developed to identify those patients with alterations in 5-FU metabolism that can lead to undue and unexpected toxicities. We previously demonstrated that the evaluation of dihydropyrimidine dehydrogenase (DPYD) polymorphisms and the 5-FU degradation rate (5FUDR) can help to distinguish patients prone to develop severe side effects in colorectal and gastric patients treated with $5-\mathrm{FU}$ in both adjuvant and metastatic setting. ${ }^{17}$

The aim of our study is to explore the association between 5FUDR, DPYD, thymidylate synthase enhancer region (TSER) and methylenetetrahydrofolate reductase (MTHFR) polymorphisms and toxicities in breast cancer patients treated with capecitabine.

\section{Patients and methods}

\section{Patients}

Clinical data of 37 patients treated for locally advanced and metastatic breast cancer at our institutions were retrospectively collected. Inclusion criteria were: age $>18$ years old, diagnosis of metastatic breast cancer, previous treatment with capecitabine for metastatic breast cancer, PS 0-1 at baseline, absence of liver or kidney impairment. Exclusion criteria were: PS 2 or more at baseline, less than one month of treatment with capecitabine and patient lost at follow up during treatment with capecitabine. As a clinical practice, CAPE was used at $1000-1250 \mathrm{mg} / \mathrm{mq}$ twice daily for 14 days followed by a seven-day rest period. We performed a monthly assessment of treatment toxicity according to the National Cancer Institute-Common Terminology Criteria for Adverse Events version 5 (CTCAE v.5, 2017). Patients were instructed to report and were usually asked for common toxicities during follow-up visits. Total toxicity was defined as the percentage of patients who suffered from at least one adverse event irrespective of type and grade. The study was conducted in accordance with the Declaration of Helsinki and the protocol approved by the institutional (Sapienza University) ethical committee.

\section{Methods}

Peripheral blood samples from all patients enrolled were collected at the baseline as clinical practice in our Institution to perform genotype analysis and evaluate 5FUDR on peripheral blood cells. Genotyping of DPYD GIVS14A (rs3918290), MTHFR C677T (rs1801133) and A1298C (rs1801131) SNPs was performed by pyro-sequencing technology. PCR analysis was used for genotyping TSER polymorphism (rs34743033). The individual 5FUDR was assessed by a liquid chromatography-tandem mass spectrometry on peripheral blood mononuclear cells (PBMC). $5 F U D R$ is determined in vitro by measuring the decrease of a fixed amount of 5-FU $(10 \mu \mathrm{g} / \mathrm{mL})$ added to a solution of PBMC, after $2 \mathrm{~h}$ incubation, expressed as nanogram per millilitre of 5-FU degraded per minute $\times 10$ cells. The assay is composed of three steps: (1) PBMC isolation from peripheral blood, (2) 
PBMC incubation with 5-FU in vitro and (3) determination of 5-FU amount to calculate the degradation rate. $^{18}$ 5FUDR is the result of the whole intracellular metabolizing process, regardless the presence or not of a single enzyme alterations. As previously reported, patients were categorized in three groups according to their 5FUDR value: below the fifth centile (poor metabolizers-PMs), above the 95th centile (ultra-rapid metabolizers-UMs) and within the 5-95th centile (0.85$2.2 \mathrm{ng} / \mathrm{mL} / 106$ cells/min) (extensive metabolizers-EM).

\section{Statistical analysis}

SPSS statistical software, Version 24 (SPSS Inc. Chicago, Illinois, USA), was used. Each MTHFR genotypes C677T (CC, CT and TT) and A1298C (AA, AC, CC), TSER genotypes $(2 \mathrm{R} / 2 \mathrm{R}, 2 \mathrm{R} / 3 \mathrm{R}, 3 \mathrm{R} / 3 \mathrm{R})$ and DPYD $(\mathrm{GG} / \mathrm{GA})$ were tested. The $\chi^{2}$ test and $\mathrm{t}$ test for unpaired data were applied to compare the frequencies and mean, respectively. Genotype variant association with toxicity events was first analysed using univariate logistic regression and further by a multivariate logistic regression including patient age ( $\geq 60$ vs. $<60$ years old), comorbidity ( $\geq 2$ vs. $<2)$ and type of chemotherapy regimen (CAPE alone vs. CAPE plus navelbine/lapatinib). A $\mathrm{P}$ value $<0.05$ was considered as statistically significant.

\section{Results}

Overall, 37 Caucasian patients with a median age of 58 years old (range 34-79) treated with CAPE-based chemotherapy for stage II-IV breast cancer were included in this study. Their baseline and demographic characteristics are shown in Table 1. A 25\% dose reduction was done in nine cases $(24.3 \%)$, and the treatment was prematurely stopped in six $(16.2 \%)$ patients due to G1-4 GI (40\%) and haematological $(60 \%)$ adverse events. The most common treatmentrelated adverse events are reported in Figure 1. CAPE was administered with an adjuvant intent in $16(43.2 \%)$ patients and in $21(56.8 \%)$ patients affected by $\mathrm{ABC}$, and it was administered alone or in combination with other drugs in $15(40.5 \%)$ and $22(59.5 \%)$ cases, respectively. Overall, $34(91.9 \%)$ patients suffered from at least an episode of any grade toxicity while $9(24.3 \%)$ patients had G3-4 toxicity. No toxic death was reported. The frequency of toxicity (73.7 vs. $77.3 \%$ and 26.7 vs. $22.7 \%$ for any grade and G3-4 toxicity, respectively) did not differ between patients who received CAPE alone or in combination with other drugs. Besides, capecitabine plus navelbine regimen showed a higher incidence of any grade haematological toxicity and G1-2 GI toxicity than capecitabine alone or in combination with lapatinib (Figure 2).
Table I. Clinicopathological parameters of patients.

\begin{tabular}{|c|c|c|}
\hline Parameter & Number & $\%$ \\
\hline Total & 37 & 100 \\
\hline Median age years (range) & $58(34-79)$ & \\
\hline \multicolumn{3}{|l|}{ Comorbidity } \\
\hline$<2$ & 32 & 86.5 \\
\hline$\geq 2$ & 5 & 13.5 \\
\hline \multicolumn{3}{|l|}{ Stage } \\
\hline II-III & 16 & 43.2 \\
\hline IV & 21 & 56.8 \\
\hline \multicolumn{3}{|l|}{ Estrogen receptor } \\
\hline Median (range) & $80(0-100)$ & \\
\hline Negative & 11 & 30.6 \\
\hline \multicolumn{3}{|l|}{ Progesteron receptor } \\
\hline Median (range) & $49.5(0-90)$ & \\
\hline Negative & 10 & 27.8 \\
\hline \multicolumn{3}{|l|}{ Her2/neu } \\
\hline Positive & 10 & 27.0 \\
\hline Negative & 27 & 73.0 \\
\hline \multicolumn{3}{|l|}{ Grading } \\
\hline I & 2 & 5.4 \\
\hline 2 & 6 & 16.2 \\
\hline 3 & 22 & 59.5 \\
\hline Missing & 7 & 18.9 \\
\hline \multicolumn{3}{|l|}{ Ki 67 expression } \\
\hline Median (range) & $32(7-89)$ & \\
\hline \multicolumn{3}{|l|}{ Capecitabine-based therapy } \\
\hline Mono-chemotherapy & 15 & 40.5 \\
\hline Plus navelbine & 17 & 46.0 \\
\hline Plus lapatinib & 5 & 13.5 \\
\hline \multicolumn{3}{|l|}{ TSER } \\
\hline $2 R / 2 R$ & 7 & 18.9 \\
\hline $3 R / 3 R$ & 14 & 37.8 \\
\hline $2 R / 3 R$ & 14 & 37.8 \\
\hline Missing & 2 & 5.4 \\
\hline \multicolumn{3}{|l|}{ MTHFR 677} \\
\hline $\mathrm{CC}$ & II & 29.7 \\
\hline CT & 19 & 51.4 \\
\hline $\mathrm{TT}$ & 6 & 16.2 \\
\hline Missing & $\mathrm{I}$ & 2.7 \\
\hline \multicolumn{3}{|l|}{ MTHFR I298 } \\
\hline $\mathrm{AA}$ & 17 & 45.9 \\
\hline$A C$ & 15 & 40.5 \\
\hline $\mathrm{CC}$ & 5 & 13.5 \\
\hline \multicolumn{3}{|l|}{ 5FU degradation value } \\
\hline EM & 31 & 83.8 \\
\hline PM & 3 & 8.1 \\
\hline UP & 3 & 8.1 \\
\hline
\end{tabular}

5FU: 5-fluorouracil; EM: extensive metabolizer; PM: poor metabolizer, UM: ultra-rapid metabolizer.

\section{Pharmacogenetic variant analyses}

The distribution of the analysed genotypes did not deviate from Hardy-Weinberg equilibrium (DPYD, $\mathrm{P}=0.9, \quad$ MTHFR 677, $\mathrm{P}=0.86 ;$ MTHFR 1298, $\mathrm{P}=0.50 ; \quad$ TSER,$\quad \mathrm{P}=0.87) . \quad$ Homozygous $\quad$ DPYD 


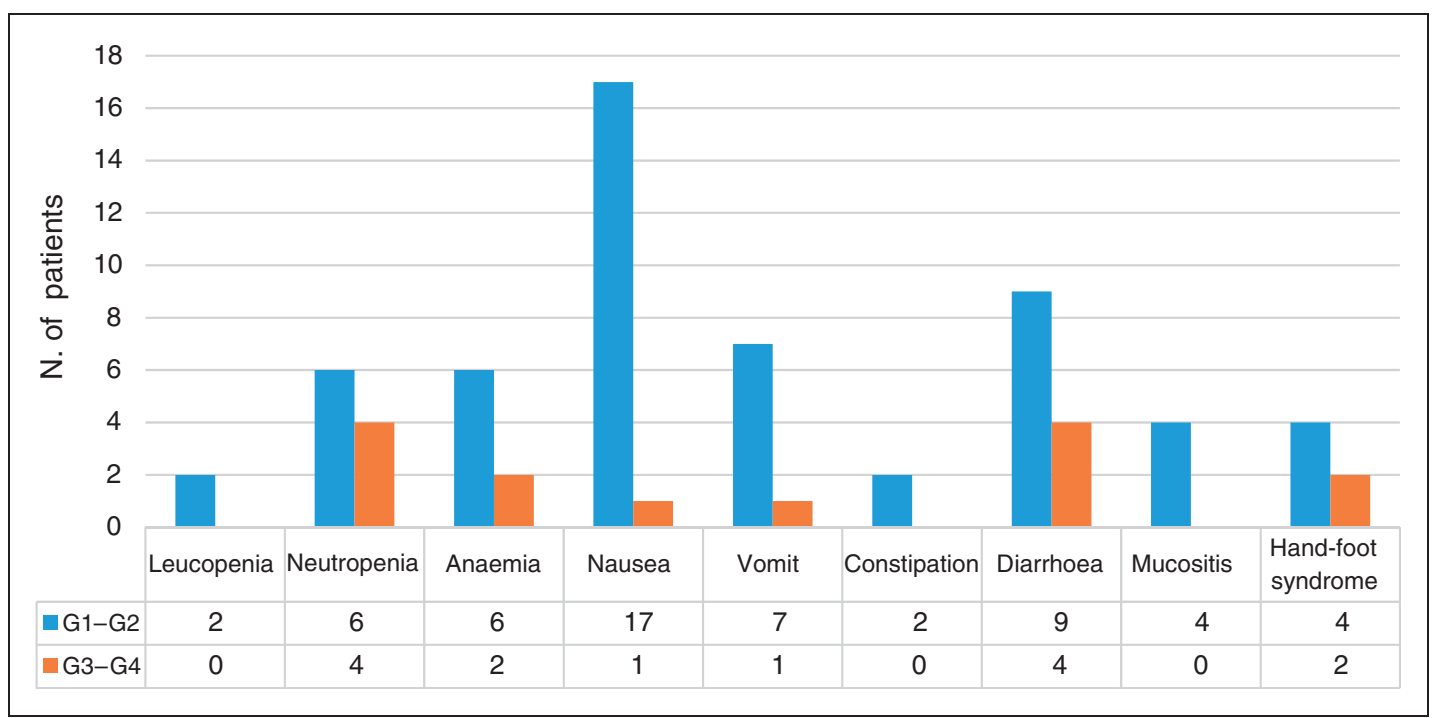

Figure I. Most common toxicities in study population.

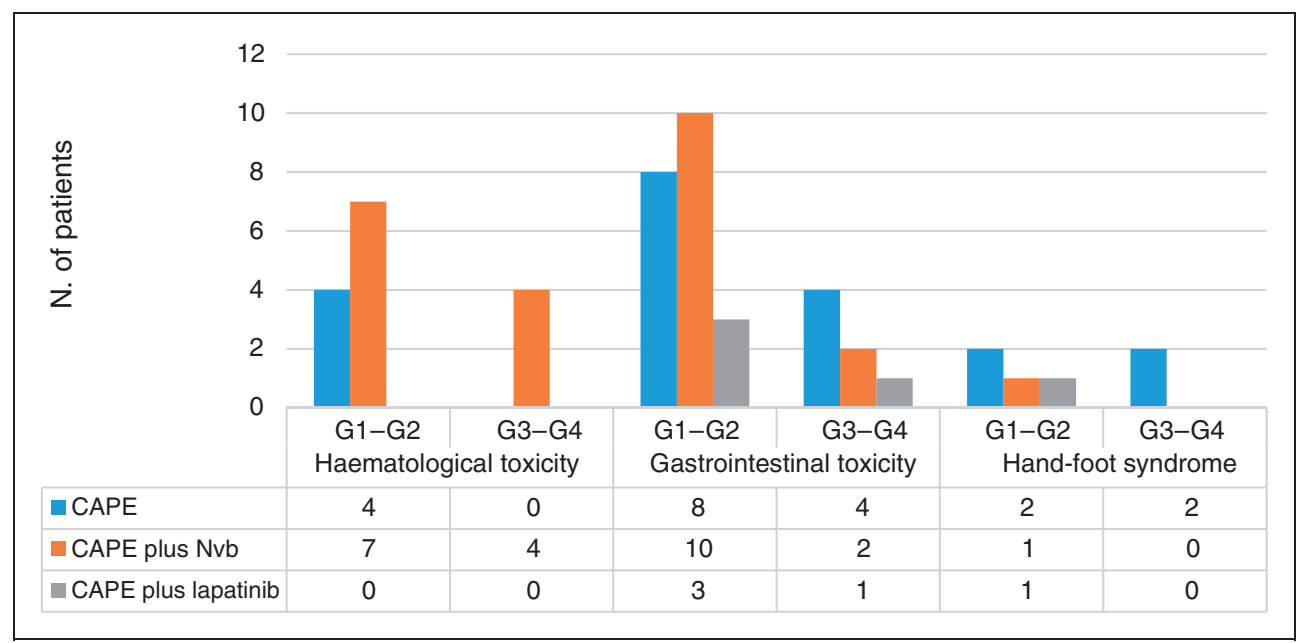

Figure 2. Type and severity of toxicity according to the therapeutic scheme administered.

IVS14 $+1 \mathrm{G}>\mathrm{A}$ SNP nor heterozygous DPYD was not observed in the cohort. Homozygous MTHFR 677TT was found to be significantly related to haematological toxicity $(\mathrm{OR}=6.5 \quad[95 \%$ IC $1.1-37.5]$, $\mathrm{P}=0.04)$. However, no association was detected between each other SNPs and toxicity (Table 2). Overall, the mean value $\pm \mathrm{SD}$ of 5 FUDR was 1.45 \pm 0.45 (range: $0.49-2.50) \mathrm{ng} / \mathrm{mL} / 106$ cells $/ \mathrm{min}$. Three patients had a degradation rate less than $0.86 \mathrm{ng} / \mathrm{mL} /$ 106 cells $/ \mathrm{min}$ (PMs) and three patients greater than $2.1 \mathrm{ng} / \mathrm{mL} / 106$ cells $/ \mathrm{min}$ (UMs). No association was found between 5FUDR and either TSER or MTHFR genotypes (Table 3). At a univariate logistic regression analysis, an altered value of 5FUDR (values $<0.86$ or $>2.10 \mathrm{ng} / \mathrm{mL} / 106$ cells $/ \mathrm{min}$ ) increased the risk of G3-4 adverse events $\quad(\mathrm{OR}=10.40 \quad$ [95\% $\quad \mathrm{IC}:$ 1.48-7.99],
$\mathrm{P}=0.02$ ) (Table 2). A multivariate logistic regression analysis, adjusted for age, comorbidity and CAPE regimen, confirmed the role of 5FUDR as a predictor of G3-4 toxicity occurrence (OR $=10.9$ [95\% IC $1.2-$ 96.2], $\mathrm{P}=0.03$.

\section{Discussion}

Several enzymes are involved in the capecitabine metabolism. ${ }^{19}$ The dihydropyrimidine dehydrogenase enzyme (DPD) metabolizes about $80 \%$ of the administered 5-FU into the inactive metabolite 5,6-dihydro-5fluorouracil. The remaining $20 \%$ is converted into active metabolites that cause the inhibition of thymidylate synthase (TYMS) and RNA/DNA damage. ${ }^{20}$ Several genotypes of the DPD have been associated 
with reduced enzyme activity that could lead to severe toxic adverse events of capecitabine or fluoropyrimidine. ${ }^{21}$ The most used pharmacogenetic test to predict DPD activity is based on the detection of IVS14+ $1 \mathrm{G}>\mathrm{A}$ polymorphism in the DPYD gene, which leads to the production of an inactive protein and severe toxicity in about one-half of carrier patients. ${ }^{22}$ Moreover, a decreased value of 5FUDR is linked to DPYD haplotype, and it could be related to adverse events development; ${ }^{23}$ however, this polymorphism has a low frequency. Other enzymes are involved in 5-FU metabolism, and their polymorphisms could result in increased and unexpected toxicities such as MTHFR, one of the most relevant enzyme that regulates intracellular folate levels that affect DNA synthesis and methylation and TYMS. ${ }^{24,25}$

The single-nucleotide polymorphisms (SNPs) of MTHFR $677 \mathrm{C}>\mathrm{T}$ and $1298 \mathrm{~A}>\mathrm{C}$ are clinically

Table 2. 5FUDR descriptive statistics by demographic and genetic characteristics.

\begin{tabular}{lrll}
\hline & $N$ & $\begin{array}{l}\text { 5FUDR } \\
\text { (mean value } \pm \text { SD) }\end{array}$ & $P$ \\
\hline Age & & & \\
$\quad<60$ years & 19 & $1.39 \pm 0.5 \mathrm{I}$ & 0.26 \\
$\begin{array}{l}\text { (m0 years } \\
\text { MTHFR C677T }\end{array}$ & 18 & $1.56 \pm 0.37$ & \\
CC & 11 & $1.56 \pm 0.63$ & 0.72 \\
CT & 19 & $1.42 \pm 0.39$ & \\
TT & 6 & $1.48 \pm 0.28$ & \\
MTHFR AI298C & & & \\
AA & 17 & $1.57 \pm 0.45$ & \\
AC & 15 & $1.47 \pm 0.36$ & \\
CC & 5 & $1.12 \pm 0.57$ & \\
TSER & & & \\
3R3R & 7 & $1.30 \pm 0.47$ & \\
2R3R & 14 & $1.51 \pm 0.41$ & \\
2R2R & 14 & $1.46 \pm 0.43$ & \\
\hline
\end{tabular}

relevant and have been associated with the toxicity of 5-FU. ${ }^{26}$ Moreover, variations of the TSER in the promoter of TYMS gene have been related to both survival/response outcomes and toxicities in patients affected by colorectal cancer treated with 5-FU-based chemotherapy. ${ }^{27-30}$ Finally, we previously described a non-genomic assay that seems to be able to predict 5-FU toxicity by the assessment of the 5FUDR in the PBMC.$^{31}$ This parameter indicates the amount of drug consumed by cells in a time unit and reflexes the result of the entire 5-FU degradation metabolism, not only a single enzyme activity. ${ }^{32}$ Applying the assay on colorectal patients, we previously described two different classes of patients with a higher risk to develop 5-FU unexpected toxicities: poor metabolizers and ultrarapid metabolizers. ${ }^{33,34}$ Besides, we showed that 5FUDR is associated with progression-free survival in metastatic colorectal patients with an advantage for ultra/poor metabolizers versus normal metabolizers. ${ }^{35}$

Up to now, no data were available on the correlation between 5FUDR and DPD/MTHFR/TSER polymorphism and capecitabine-related toxicity specifically in breast cancer patients. Hence, we carried out a retrospective study aimed to evaluate the impact of each of the following gene polymorphisms MTHFR C667T, MTHFR A1298C, DPYD IVS14 + 1G > A, TSER and the 5-fluorouracil degradation rate (5-FUDR) on toxicities in breast cancer patients treated with capecitabine. Our hypothesis was that ultra/poor metabolizer patients have a higher percentage of total adverse events.

Our results suggest that 5FUDR is a possible predictor of G3-4 toxicity in both metastatic and non-metastatic breast cancer patients treated with capecitabine. UMs and PMs patients developed higher rate of severe toxicities compared with NM, and these results are similar to our previous findings on colorectal cancer patients treated with capecitabine,

Table 3. Incidence of toxicity by patients genotype and 5FUDR.

\begin{tabular}{|c|c|c|c|c|c|c|c|c|c|c|c|c|c|c|}
\hline Biomarker & Genotype & $N$ & $\begin{array}{l}\text { Haematoxicity } \\
(\text { GI-4) } \\
(\%)\end{array}$ & $\begin{array}{l}\text { OR } \\
(95 \% \mathrm{Cl})\end{array}$ & $P$ & $\begin{array}{l}\text { GI toxicity } \\
(\mathrm{GI}-4) \\
(\%)\end{array}$ & $\begin{array}{l}\text { OR } \\
(95 \% \mathrm{Cl})\end{array}$ & $P$ & $\begin{array}{l}\text { HFS } \\
(\mathrm{GI}-4) \\
(\%)\end{array}$ & $\begin{array}{l}\text { OR } \\
(95 \% \mathrm{Cl})\end{array}$ & $P$ & $\begin{array}{l}\text { G3-4 } \\
\text { Toxicity } \\
\text { (\%) }\end{array}$ & $\begin{array}{l}\text { OR } \\
(95 \% \mathrm{Cl})\end{array}$ & $P$ \\
\hline MTHFR & $\mathrm{CC}$ & 11 & 18 & & & 91 & & & 35 & & & 36 & & \\
\hline \multirow{2}{*}{ C677T } & CT & 16 & 56 & & & 75 & & & 6 & & & 21 & & \\
\hline & TT & 6 & 66 & $6.5(1.1-37.5)$ & 0.04 & 83 & $0.3(0.1-3.3)$ & 0.35 & 20 & $0.2(0.1-1.2)$ & 0.08 & 17 & $0.4(0.1-2.1)$ & 0.30 \\
\hline \multirow{3}{*}{$\begin{array}{l}\text { MTHFR } \\
\text { AI298C }\end{array}$} & AA & 15 & 53 & & & 73 & & & 21 & & & 29 & & \\
\hline & $A C$ & 14 & 43 & & & 85 & & & 7 & & & 13 & & \\
\hline & $\mathrm{CC}$ & 5 & 20 & $0.5(0.1-2.0)$ & 0.33 & 100 & $3.1(0.5-19.8)$ & 0.23 & 40 & $0.7(0.1-4.0)$ & 0.67 & 40 & $0.6(0.1-2.7)$ & 0.50 \\
\hline \multirow[t]{3}{*}{ TSER } & $3 R / 3 R$ & 7 & 43 & & & 86 & & & 0 & & & 29 & & \\
\hline & $2 R / 3 R$ & 12 & 42 & & & 83 & & & 25 & & & 29 & & \\
\hline & $2 R / 2 R$ & 13 & 54 & $1.2(0.2-6.6)$ & $0.8 \mathrm{I}$ & 77 & $0.6(0.1-6.8)$ & 0.73 & 23 & $1.5(0.2-8.9)$ & 0.65 & 14 & $0.7(0.1-4.4)$ & 0.68 \\
\hline \multirow{3}{*}{$\begin{array}{l}\text { 5-FU } \\
\quad \text { dRate }\end{array}$} & NM & 28 & 43 & $1.3(0.2-7.8)$ & & 86 & & & 22 & & & 16 & $10.4(1.5-72.9)$ & \\
\hline & PM & 3 & 33 & & & 67 & $0.3(0.1-2.4)$ & & 0 & & & 67 & & \\
\hline & UM & 3 & 67 & & 0.75 & 67 & & 0.28 & 0 & 0.0 & 0.99 & 67 & & 0.02 \\
\hline
\end{tabular}

Bold- significant difference in G3-4 toxicities between normal metabolizers (NM) and ultra/poor metabolizers (PM/UM) $(p=0.02)$. 
suggesting a predictive value of 5FU-degradation regardless of cancer origin and stage.

Moreover, as previously described, 5FUdegradation rate can evaluate the result of the whole intracellular metabolism of 5FU, regardless the single enzymatic alteration. Indeed, some of the most used pharmacogenetic assays to predict DPD activity evaluate the presence of the splice site IVS14+1G $>$ A polymorphism in the DPYD gene, but this alteration has a low frequency, and it is not present in some of the patients with severe toxicity. About that, in our limited population, no patient with DPYD alteration has been identified even in the presence of patients with severe toxicities who need dose reduction. Finally, 5FUDR is faster, results can be obtained in few days, and cheaper, the cost is less than $€ 10$ per patient, if compared with genotyping.

The test could be suggested in particular to women that should start an adjuvant treatment next to a noncomplete pathological response to neoadjuvant chemotherapy in triple negative disease. In the adjuvant setting, a test able to predict severe toxicities in these particular setting is crucial in order to avoid reduced dose, delayed administration or interruption of therapy and to maintain dose intensity. Indeed, in metastatic setting, the prediction of severe toxicities could help the clinicians to choose the best treatment to improve the quality of life.

Finally, our study has some relevant limitations: it is a retrospective and monocentric study and the population is limited. Our findings should be confirmed in a prospective study and on a larger breast cancer patient's sample to extend the use of 5FUDR in clinical practice.

Despite the limitations of our retrospective study, we confirm the results showed in other cancer subtypes. We highlight the importance of conducting prospective studies on larger sample size and on a homogeneous population in order to evaluate the 5FUDR impact on both toxicities and outcome.

\section{Conclusion}

The pre-treatment evaluation of 5-FUDR allows to identify breast cancer patients at high risk for severe 5-FU toxicity such as in colorectal and gastric patients cohort.

\section{Author's Note}

Andrea Botticelli is now affiliated to Department of Clinical and Molecular Medicine, Sapienza University of Rome, Rome, Italy And Simone Scagnoli is now affiliated to department of medical surgical science and translational medicine, Sapienza University of Rome, Rome, Italy.

\section{Declaration of Conflicting Interests}

The author(s) declared no potential conflicts of interest with respect to the research, authorship, and/or publication of this article.

\section{Funding}

The author(s) received no financial support for the research, authorship, and/or publication of this article.

\section{ORCID iD}

Simone Scagnoli (DD https://orcid.org/0000-0003-4943-5622

\section{References}

1. Miwa M, et al. Design of a novel oral fluoropyrimidine carbamate, capecitabine, which generates 5 fluorouracil selectively in tumours by enzymes concentrated in human liver and cancer tissue. Eur J Cancer 1998; 34: 1274-1281.

2. Scheller J, et al. Preferential activation of capecitabine in tumor following oral administration to colorectal cancer patients. Cancer Chemother Pharmacol 2000; 45: 291-297.

3. Pfeiffer $P$, et al. Patient preference for oral or intravenous chemotherapy: a randomised cross-over trial comparing capecitabine and Nordic fluorouracil/leucovorin in patients with colorectal cancer. Eur J Cancer 2006; 42: 2738-2743.

4. Blum JL, et al. Multicenter, phase II study of capecitabine in taxane-pretreated metastatic breast carcinoma patients. Cancer 2001; 92: 1759-1768.

5. Mavroudis D, et al. Randomized phase III trial comparing docetaxel plus epirubicin versus docetaxel plus capecitabine as first-line treatment in women with advanced breast cancer. Ann Oncol 2009; 21: 48-54.

6. Bajetta E, et al. Safety and efficacy of two different doses of capecitabine in the treatment of advanced breast cancer in older women. J Clin Oncol 2005; 23: 2155-2161.

7. Geyer CE, et al. Lapatinib plus capecitabine for HER2positive advanced breast cancer. $N$ Engl J Med 2006; 355 : 2733-2743.

8. Martín M, et al. Epirubicin plus cyclophosphamide followed by docetaxel versus epirubicin plus docetaxel followed by capecitabine as adjuvant therapy for nodepositive early breast cancer: results from the GEICAM/ 2003-10 study. J Clin Oncol 2015; 33: 3788-3795.

9. Joensuu H, et al. Adjuvant capecitabine in combination with docetaxel and cyclophosphamide plus epirubicin for breast cancer: an open-label, randomised controlled trial. Lancet Oncol 2009; 10: 1145-1151.

10. Masuda N, et al. Adjuvant capecitabine for breast cancer after preoperative chemotherapy. $N$ Engl J Med 2017; 376: 2147-2159.

11. Ma Y, et al. Capecitabine for the treatment for advanced gastric cancer: efficacy, safety and ethnicity. J Clin Pharm Therapeut 2012; 37: 266-275.

12. Haller DG, et al. Potential regional differences for the tolerability profiles of fluoropyrimidines. J Clin Oncol 2008; 26: 2118-2123. 
13. Leicher LW, de Graaf JC, Coers W, et al. Tolerability of capecitabine monotherapy in metastatic colorectal cancer: a real-world study. Drugs $R$ D 2017; 17: 117-124.

14. Mikhail SE, Sun JF and Marshall JL. Safety of capecitabine: a review. Expert Opin Drug Saf 2010; 9: 831-841.

15. Stintzing $\mathrm{S}$, et al. Correlation of capecitabine-induced skin toxicity with treatment efficacy in patients with metastatic colorectal cancer: results from the German AIO KRK-0104 trial. Br J Cancer 2011; 105: 206-211.

16. Sendur MAN and Kilickap S. Stevens-Johnson syndrome after treatment with capecitabine. Clin Oncol 2008; 20: 202-203.

17. Onesti CE, et al. 5-Fluorouracil degradation rate could predict toxicity in stages II-III colorectal cancer patients undergoing adjuvant FOLFOX. Anticancer Drug 2016; 28: $322-326$.

18. Lostia AM, et al. A liquid chromatography-tandem mass spectrometry method for the determination of 5-fluorouracil degradation rate by intact peripheral blood mononuclear cells. Ther Drug Monit 2009; 31: 482-488.

19. Thorn CF, et al. Pharm GKB summary: fluoropyrimidine pathways. Pharmacogenet Genom 2011; 21: 237-242.

20. Longley DB, Harkin DP and Johnston PG. 5-Fluorouracil: mechanisms of action and clinical strategies. Nat Rev Cancer 2003; 3: 330-338.

21. Stavraka C, et al. Clinical implementation of pretreatment DPYD genotyping in capecitabine-treated metastatic breast cancer patients. Breast Cancer Res Treat 2019; 175: 511-517.

22. Schwab M, et al. Role of genetic and nongenetic factors for fluorouracil treatment-related severe toxicity: a prospective clinical trial by the German 5-FU toxicity study group. J Clin Oncol 2008; 26: 2131-2138.

23. Gentile G, et al. Genotype-phenotype correlations in 5-fluorouracil metabolism: a candidate DPYD haplotype to improve toxicity prediction. Pharmacogenom J 2016; 16: $320-325$.

24. Etienne-Grimaldi MC, et al. Methylenetetrahydrofolate reductase (MTHFR) gene polymorphisms and FOLFOX response in colorectal cancer patients. $\mathrm{Br} \quad \mathrm{J}$ Clin Pharmacol 2010; 69: 58-66.
25. Sharma R, et al. Thymidylate synthase and methylenetetrahydrofolate reductase gene polymorphisms and toxicity to capecitabine in advanced colorectal cancer patients. Clin Cancer Res 2008; 14: 817-825.

26. Thomas F, et al. Methylenetetrahydrofolate reductase genetic polymorphisms and toxicity to 5-FU-based chemoradiation in rectal cancer. $B r J$ Cancer 2011; 105: 1654-1662.

27. Martinez-Balibrea E, et al. UGT1A and TYMS genetic variants predict toxicity and response of colorectal cancer patients treated with first-line irinotecan and fluorouracil combination therapy. Br J Cancer 2010; 103: 581-589.

28. Pander $\mathbf{J}$, et al. Pharmacogenetic interaction analysis for the efficacy of systemic treatment in metastatic colorectal cancer. Ann Oncol 2011; 22: 1147-1153.

29. Pullarkat ST and Lenz HJ. Thymidylate synthase gene polymorphism determines response and toxicity of 5-FU chemotherapy. Pharmacogenom J 2001; 1: 65-70.

30. Romiti A, et al. The TYMS-TSER polymorphism is associated with toxicity of low-dose capecitabine in patients with advanced gastrointestinal cancer. Anticancer Drugs 2016; 27: 1044-1049.

31. Roberto M, et al. Evaluation of 5-fluorouracil degradation rate and pharmacogenetic profiling to predict toxicity following adjuvant capecitabine. Eur J Clin Pharmacol 2017; 73: 157-164.

32. Botticelli A, et al. A nomogram to predict 5-fluorouracil toxicity: when pharmacogenomics meets the patient. Anticancer Drugs 2017; 28: 551-556.

33. Mazzuca F, et al. Pre-treatment evaluation of 5-fluorouracil degradation rate: association of poor and ultra-rapid metabolism with severe toxicity in a colorectal cancer patients cohort. Oncotarget 2016; 7: 20612-20620.

34. Simmaco M, et al. Pre-treatment assay of 5-fluorouracil degradation rate (5-FUDR) to improve prediction of 5-fluorouracil toxicity in gastro-esophageal cancer. Oncotarget 2016; 8: 14050-14057.

35. Botticelli A, et al. Degradation rate of 5-fluorouracil in metastatic colorectal cancer: a new predictive outcome biomarker? PLoS One 2016; 11: 0163105. 